\title{
Blockchains as Enablers for Auditing Cooperative Circular Economy Networks
}

\author{
George Alexandris, Vassilis Katos \\ Dept. of Computing and Informatics \\ Bournemouth University \\ Bournemouth, United Kingdom \\ galexandris@bournemouth.ac.uk, \\ vkatos@bournemouth.ac.uk
}

\author{
Sofia Alexaki \\ Ecole des Ponts Business School \\ Paris, France \\ s.alexaki@pontsbschool.com
}

\author{
George Hatzivasilis \\ Institute of Computer Science \\ Foundation for Research and \\ Technology-Hellas (FORTH) \\ Heraklion, Crete, Greece \\ hatzivas@ics.forth.gr
}

\begin{abstract}
Implementing a circular economy business model which is profitable for businesses operating physical assets, while at the same time does not conflict with strategic goals of environmental policies can be a complex and risky undertaking for a single entity, especially if the asset operator is a small-to-medium enterprise (SMEs). To mitigate this, a collaborative circular economy business model is proposed, where the circular economy cycle is materialized by assets transitioning between asset operators on a demanddriven approach. Demand itself is partially based on the asset's state, which is described by its circular properties (location, condition, availability). The asset state and its transition between operators can be monitored by auditors and governmental regulators to ensure asset integrity and compliance with environmental targets. This common view of asset state between all parties can be enabled by blockchains and smart contracts, which can provide the underlying technology to share data with integrity, while simultaneously offering more efficient interoperability between participants. To demonstrate how this could be achieved, a conceptual asset record access and sharing mechanism is presented which is suitable for regulated environmental jurisdictions.
\end{abstract}

Keywords-Circular Economy, Business Models, Blockchains, Smart Contracts

\section{INTRODUCTION}

Circular Economy characterizes an economy that is restorative and regenerative by design, aiming to keep products, components and materials at their highest utility and value at all times. Its value drivers include extending the useful life of finite resources, maximising the utilisation of assets and creating new use cycles for end-of-life assets. According to [1], a circular economy could generate net economic gains of E1.8 trillion per year by 2030 . Key value drivers for a circular economy are extending its use cycle length, increasing its utilization, looping the asset though additional use cycles and regeneration of natural capital [2][3]. Pairing the circular economy model with the advancement of digital technologies has sparked a fast growing area of research [4], focusing on how data derived from various sources can be leveraged to create a circular product life-cycle. Following a systematic literature research, Pagoropoulos et al have identified three major areas where digital technologies can be applied to the Circular Economy, namely collection, analysis and integration of data [5]. Within the area of data collection, the Internet of Things (IoT) domain plays a prominent role, given that the information collected by sensors for their underlying assets, can map directly to the attributes location, condition and availability, which, according to [6] form the fundamental for circularity. Consequently, IoT-enabled assets can leverage their intrinsic data collection capabilities to act as key enablers for the circular economy, as proposed by [7]. However, Panagopoulos et al conclude that, although the interplay between the aforementioned areas of data collection, data analysis and data integration is well understood, in the current scientific literature a "limited technological perspective" exists with regards to applying these technologies to Circular Economy use cases. This confirms Lieder and Rashid [8], who state that most studies neglect business and economic perspectives. With the above in mind, the present study attempts to tackle this shortcoming by proposing a concrete application of a collaborative circular economy network based on asset value drivers and leveraging the recent advances in blockchain technology.

The paper is structured as follows. Section 2 offers a brief overview of circular economy business models and how they are understood within the scope of this work. Section 3 presents the concept of the application, how it relates to the circular economy cycle and, to a degree, addresses shortcomings of existing circular economy business models. Section 4 describes the technical aspects of the application and the underlying blockchain environment. Section 5 suggests application enhancements, future research topics and concludes the proposal.

\section{CIRCULAR ECONOMY BUSINESS MODELS}

There are several studies in the literature on how a Circular Economy Business Model (CBM) is defined, and what qualifies a conventional business model to be labelled as 'circular'. Linder and Williander [9] define CBMs as models in which "the conceptual logic for value creation is based on utilizing the economic value retained in products after use in the production of new offerings" In our opinion, this statement mirrors the economic incentives of businesses, given that it shifts the focus of CBMs to value creation; it also implies that for businesses, social and ecological sustainability goals are better served at a macro-level. This is also in line with Lewandowski in [10], who states that the core component of a circular business model clearly is its value proposition. Several frameworks have been proposed as to how generation of value can occur in circular economy, including McKinsey\&Company's ReSOLVE framework [11] and Laubscher and Marinelli's six key areas of integrating the CE 
concepts in existing business processes [12]. The ReSOLVE framework consist of six action areas to apply the $\mathrm{CE}$ principles: 'Regeneration', 'Share', 'Optimise', 'Loop', ' $V$ irtualise' and 'Exchange'. A detailed description of each area would be out of scope of this work; for the purposes of our application, 'Share', 'Optimise' and 'Loop' are of importance. 'Share' refers to keeping the product loop speed low and maximizing product utilization by sharing or reusing. 'Optimize' means increasing product performance/efficiency by leveraging product data provided by, among other sources, IoT sensors. Finally, 'Loop' aims to keep products and their constituting materials in closed loops, i.e. performing necessary actions to loop the product or its materials through another life cycle. Similar to the ReSOLVE framework, Laubscher and Martinelli's perspective for applying CE principles to business processes also include 'Supply Loops', an area geared towards maximizing recovery of assets and materials, and 'IT/Data Management', which focuses on optimizing resources by keeping track of products and material data. Another area which is of relevance in our proposed application is 'Strategic sourcing for own operations', which aims to build trusted partnerships and long-term relationships with suppliers and customers.

When researching business models, the components of a business model (as discussed above), are only one of the eight sub-domains described by Pateli and Giaglis [13]. When it comes to materializing components to concrete business processes, the domains Design Methods and Tools and Adoption Factors play an important role. Within the former domain, various studies exist [14] [15] which generally aim to articulate a procedure for small and medium enterprises (SMEs) to follow, in order to adopt and benefit from circular economy practices. One thing common to all studies is that a candidate SME needs to traverse through multiple stages to implement the mechanisms of a circular economy, ranging from five steps [16] [17], all the way to eleven stages [18]. The transition usually starts with learning the fundamental circular economy concepts, deciding on business objectives, and evaluating the readiness of the company. The next steps generally involve detailed (re-) design of company processes, including deciding on end-of-life criteria [19]. Finally, the transition concludes with implementing and supporting the $\mathrm{CE}$ processes and examining whether value has been generated.

Further, Scott [14] also proposes the introduction of environmental audits (e.g. compliance, waste etc.), which, as previously mentioned, may not be necessarily aligned with the primary business goal of value creation. The concept of auditing is encountered again in the domain of Adoption Factors, where legislation and policy makers ranging from municipal to international government levels can impact the adoption of a CBM by a business [20]. In addition to regulatory aspects, other important areas to consider within this domain are conditions to ultimately ensure profitability of a closed loop (analysed among others by Winter in [21]), suitable human resources and leadership [22], effective IT and Data Management [19] and finally, the business risk involved in validating a CBM which is always higher than its corresponding traditional linear counterpart [9].

From the research on CBMs described in this section, it becomes evident that transitioning to circular economy practices is not a trivial task. Winans et al have identified several technical and economical barriers for implementing a successful CE concept [23]. Given that the transition to $\mathrm{CE}$ principles may require substantial upfront investments in time and effort to rethink most aspects of a company's operations, it may easily overwhelm and become prohibitive for SMEs. Even elementary CE concepts like determining what happens after product end-of-life, is according to Okorie et al [4] a research area still in its infancy for manufacturers of digital technology products. More importantly, a transitioning an existing business to the $\mathrm{CE}$ mandates changing the company's product value proposition, which inherently comes attached with a nonnegligible amount of risk.

\section{APPLICATION CONCEPT}

In the author's view, realizing an effective and profitable closed-loop CE system may be daunting task for most SMEs due to the reasons laid out in the previous section. This point of view is shared also by Winkler in the context of supply chains, [24], who, while stressing the importance of closed loops, underlines that companies as a single unit cannot implement effective closed systems. Fig. 1 depicts a simplified closed cycle of a single SME asset operating owner, altering the usage pattern of an asset depending on the asset's location, condition and availability properties (LCA).

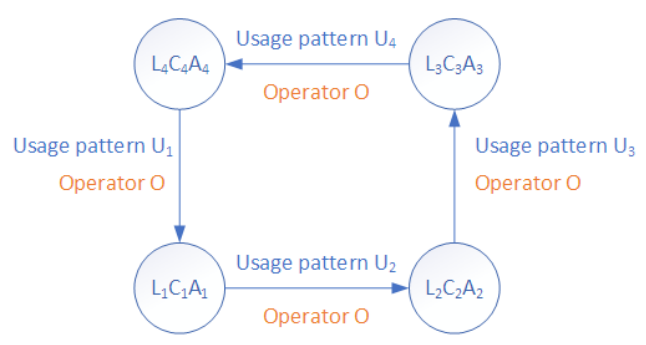

Fig. 1: Asset transition within a CE cycle for a single asset operator

The need for cooperative networks is also emphasized by [25] and [26], mentioning that circularity can hardly be achieved without collaboration. The point underpinning the application concept is that circular economy business models for SMEs will eventually rely on collaborating entities, as it is unlikely that a single SME will possess the necessary means and expertise to implement an effective and profitable business model to exploit an asset's lifecycle to its full potential. Given the above, it makes economical sense for an SME to be involved only in a particular stage of the asset lifecycle, for which it will have optimized its operations. During this stage, the business model from the perspective of the acting entity can be seen as linear, thus removing much of the complexity and risk induced by a circular business model. However, from an asset perspective the business model remains circular, albeit consisting of a sequence of linear business models performed by different entities. We refer to the time period where a single entity controls and capitalizes on an asset as a profit stage. Since the entire asset life span is no longer controlled by a single entity, neither the duration of each profit stage nor the next profit stage are known a priori. Hence we refer to this life span as an ad-hoc circular cycle which exhibits a loose coupling between profit stages. Of particular interest in an adhoc circular cycle are the transitions between profit stages. Here, the first two questions that come to mind are: 
- when the control of an asset should transition to another entity, and

- which entity should control the next profit stage of an asset.

Building on Linder and Martinelli's definition of a CBM, we use the value proposition as the driver to answer these questions. Therefore the asset should transition to the next profit stage if the expected generated value in the next potential profit stage of the asset is higher than the value it generates in its current profit stage. Accordingly, the entity which expects to extract the maximum possible value from the asset in its next profit stage should be the next to control the asset. Since the value of an asset is usually tightly coupled with its role in a system, and the controlling entity defines the role of an asset, it becomes evident that different entities may value the same asset differently, depending on how they envisage to utilize it. However, the underlying circularity properties of an asset, i.e. location, condition, availability, always need to be factored in its evaluation. Thus for an entity to calculate the expected generated value of an asset, information about its circular properties must be known, i.e. shared within a network of entities who control, or wish to control assets. Ideally, this information should always be relevant, accurate and timely, but it is fair to assume that, from the point of view of an entity which does not control an asset but is interested in controlling it, information originating solely from the entity which currently controls the asset may not be entirely trustworthy.

In addition to generating value for the controlling entities, transitioning between profit stages should also be compatible with long-term sustainability goals and the circular economy strategies as these may be formulated by policy makers [20]. In light of this, the possibility of making transitions dependent on reaching life-cycle assessment targets or certifications [27] can be imagined.

We thus introduce the concept of a cooperative circular economy network consisting of three main categories of participants who share a common platform: asset operators, auditors and regulators.

- An operator represents an entity which has controlling ownership of an asset and has a stake in operating it for profit. Assets can be exchanged between entities, with the purpose of more effectively (and profitably) utilizing an asset's LCA properties.

- A regulator represents the authority which is responsible for establishing rules governing interactions between asset operators in accordance with the macroscopic sustainability targets of the network's CE policy.

- An auditor is a third party which is entrusted by the operator to verify the asset's LCA properties, thus fostering trust between the current operator and prospective operators of the asset. Further, the auditor can certify that assets abide to CE policy goals.

The resulting ad-hoc circular cycle of an asset with the audited transition points between operators is shown in Fig. 2:

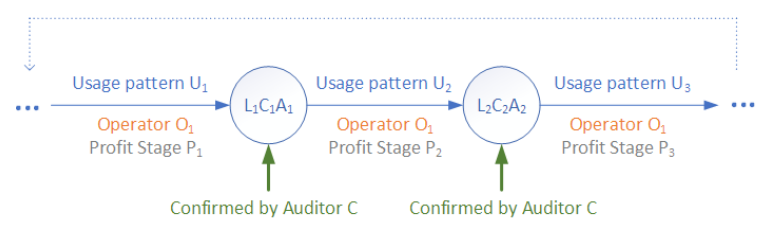

Fig. 2: Asset transition in an audited ad-hoc collaborative CE cycle

\section{APPLICATION DESCRIPTION}

A blockchain-based application can form the backbone of a decentralized $\mathrm{CE}$ platform for asset operators, prospective asset operators, auditors and regulators. The asset's LCA properties are stored on the blockchain and act as a shared resource. Several advantages of this approach are:

- All updates to an asset's state are immutably recorded, thus the complete history of asset usage can be retraced.

- No single authority has custody over the asset record.

- Increased resilience to failures or security incidents which can affect access to an asset record.

- Common view of the state of an asset's record, accessible by all participating entities.

- "Smart Contracts" residing on the blockchain and owned by the asset operator can be used to control who is authorized to perform what.

- Extendable to include other blockchain-based functions such as asset ownership exchanges or automated interactions triggered by LCA properties.

\section{A. Working Context}

Despite all benefits, due to inherent technological limitations stemming from a decentralized architecture, Blockchains cannot effectively address every possible business case. Within the scope of this work, the problem space will be constrained to the requirements of environmental policy regulators, who would maintain a jurisdiction-wide registry of assets for asset tracking and life-cycle assessment purposes. We examine the scenario where these authorities can assume an active role in overseeing asset operation with respect to meeting sustainability targets, not merely by compliance auditing "after the fact". As a prerequisite for auditing, we expect that these authorities would define the critical LCA properties to be monitored and the format used for storing them on the blockchain. Put differently, the authority can prescribe an interface which needs to be honored by all parties. With regards to asset record access management, we envisage the following basic rules:

- Asset operators should have complete access to their asset's record.

- Auditors can be entrusted by an asset operator to update all or parts of the asset record.

- Asset operators should be notified when auditors or regulators wish to access their record.

It should be noted that the above requirements could easily be met by a governmental authority acting as a gatekeeper 
and a repository for all asset records in a centralized fashion. However, this violates the principle of decentralization which is crucial in ensuring that asset operators retain full control of their asset data while building trust between operators, prospective operators and regulators. Simultaneously, single failure points can be avoided. In this light, a blockchain can help satisfy these requirements without resorting to a centralized architecture.

\section{B. Participation Considerations}

One of the main questions when designing a blockchain system is whether it should be public or permissioned. For the case of authorities governing environmentally regulated activities, identification of all participants can be considered mandatory. Therefore it makes sense to opt for a permissioned blockchain, in order to benefit from potential higher throughput as mentioned by Christidis et al in [28], given that identification of operators and auditors needs to occur anyway in order to oversee their activities. In addition to identifying the blockchain participants, the authority can decide who can run blockchain nodes and validate transactions. In our opinion, blockchain transactions could be validated by a miner network formed by the regulating authority itself, asset operators, prospective asset operators and auditors. All the aforementioned participants have an interest in viewing the current state of asset data, thus they are incentivized to maintain the decentralized network. Other participants without mining rights can include local communities and environmental NGOs. An additional benefit of using a permissioned blockchain would be that the authority retains a degree of control over source code and blockchain governance.

\section{Smart Contracts}

The main building blocks of the application are implemented using Smart Contracts, a common term to describe stored programs on the blockchain which can be run by triggering a transaction to them. Smart contracts are uniquely addressable, can preserve state and execute in a prescribed manner within the virtual machine of the blockchain. The most prominent example of Smart Contract support is the Ethereum blockchain [29], which offers a Turing-complete programming language for programming complex logic in smart contracts. Smart Contracts can evolve in decentralized autonomous organizations (DAOs), a term describing contracts calling other contracts and depending on the outcome, are able to change their behaviour based on already encoded rules [30]. Within this work, Smart Contracts are implemented for the Ethereum blockchain (or Quorum [31], its permissioned sibling) using the Solidity programming language [32]. The groundwork of the application is based on the following smart contracts which extend:

Asset Registry: A contract which contains the addresses (i.e. the public key) of all assets and maps them to their real-life identifier. Together with the asset address, the addresses of the asset's current operator and auditor are stored. This contract is owned and managed by the regulating authority, in this case a governmental entity.

Asset State Record: A contract which holds the actual state of the asset's Location-Condition-Availability properties. Every asset has an asset state contract which is owned by the asset operator. However, the asset's LCA properties can be updated either by the asset operator or by the asset auditor.

Asset Audit Agreement: This is a contract - also in the literal sense - between an asset operator and an auditor, which determines in a granular fashion what parts of the asset state contract the auditor can access. The access scope and the access conditions (e.g. time window) are described in this agreement. The contract is created and owned by the asset operator.

Asset Agreement List: A contract which can be either owned by an asset operator or by an auditor, and contains a mapping of all the contract owner's Asset Audit Agreement addresses with the target assets.

\section{Data Model}

A high-level depiction of the data model resulting from the smart contracts described above is depicted in Fig. 3 . The data rows are implemented in the Solidity

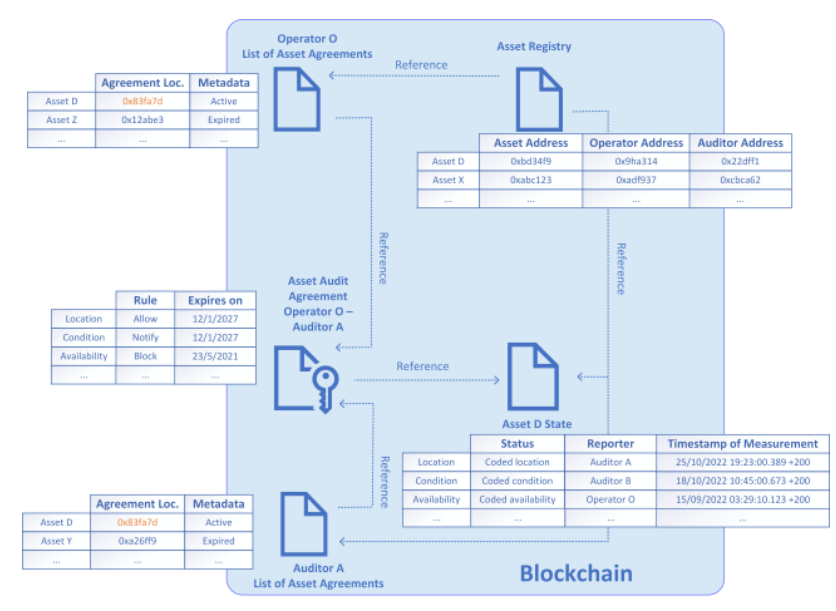

Fig. 3: Data model and relation between contracts

programming language using the mapping construct for keyvalue pairs. Contracts can reference each other's location using the address type. In the example shown below, Asset Registry references many Asset Agreement Lists, which in turn reference many Asset Audit Agreements. Finally, each Asset Audit Agreement references a single Asset State Record.

Agreements can be augmented with metadata denoting the validity of the agreement. The validity of the agreements is usually managed by the asset operator and be cancelled, renewed or set to expire automatically after a certain period, thus capturing various business cases where an operator terminates the association with the auditor. Further to the validity of an agreement, functions can be added to fine-tune permissions, and notification patterns. These functions themselves can also be subject to validity constraints, which can be embedded into the function call using the Solidity modifier type, which can be used to turn functions "on" and "off", resembling contract "termination by right" [33].

The asset location, condition and availability data is kept in the Asset State Record contract using codifications for the most recent location, condition and availability of the asset. In our design, the LCA attributes themselves act as the 
primary key for identifying a logical set of asset data, although other approaches are possible. Each set of data represents a reading on the asset and consists of the entity reporting the reading and the timestamp of the reading. As mentioned previously, activities need to be codified for minimizing storage requirements (which is important for optimal blockchain operation), but also to be universally interpretable given that this is a shared record between stakeholders of the circular ecosystem. It can be envisaged that authority governing the blockchain will enforce a common codification standard.

\section{E. Operative Cases}

The proposed mode of operation of the system is illustrated using two common use cases which are detailed in this section.

1) Creating the operator-auditor agreement for asset access

This use case is a prerequisite for all subsequent monitoring actions by a specific auditor. Through the concept of a different Asset Audit Agreement contract per auditor, the system can allow cases where different auditors monitor different parts of the asset, e.g. the location of an asset can be verified by auditors specializing in tracking assets.

The Asset Audit Agreement contract is created by the auditor, but is owned and controlled by the asset operator. In order to create the agreement, the auditor needs to look up the operator's agreement list via the Asset Registry contract and request to be added to it by calling an appropriate function in the Asset Agreement List contract. This function, when called, notifies the operator, who can accept or decline this request using an off-chain application. If the operator accepts the request, the auditor creates a new Asset Audit Agreement contract and requests from the asset operator to sign it. In signing the contract, the asset operator also updates the permissions of the Asset State contract, granting the newly created Asset Audit Agreement contract full or partial access to it. At the same time, both the asset operator's and the auditor's Asset

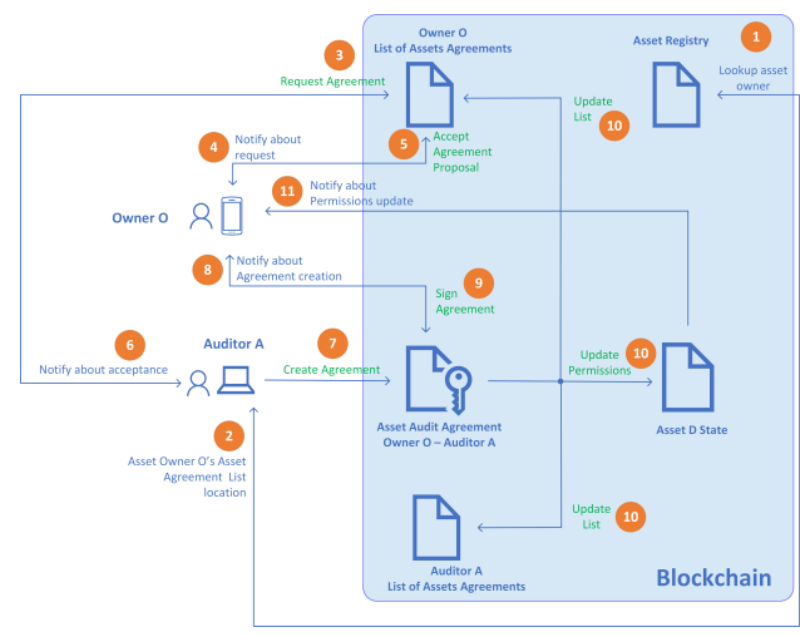

Fig. 4: Sequence required to create an operator-auditor agreement

Agreement List contracts will be updated to reflect the new access permissions. The flow of the operation is illustrated in Fig. 4. Although there are several interactions depicted, only the ones in green will alter the state of the contracts and will be recorded on the blockchain. The other transactions can be logged by off-chain applications, depending on the preferences of the users.

\section{2) Allowing an auditor to access the Asset State Record} for update

An auditor may access the asset's state record for various reasons, and depending on the auditor's intent, the agreement contract can enforce rules for allowing or prohibiting access. The access rules of the contract can be managed directly by the asset operator via an off-chain application. The same application can be notified by the agreement contract when access to the asset state record is requested.

Access to an asset's Asset State Record contract by an auditor occurs only via the Asset Audit Agreement contract. Consequently, whenever an auditor wishes to access the Asset State Record contract, the auditor should first locate the Asset Audit Agreement contract for this asset in the auditor's own Asset Agreement List contract. Once the contract is located, the auditor calls a function of the Asset Audit Agreement contract which performs the sought action with respect to the asset's state record (e.g. update the asset's condition data). After successfully validating the permissions of the caller, the function emits an event to notify the operator about the access to the asset's record and calls the appropriate function in the Asset State Record contract, which returns the requested data. Fig. 5 shows in more detail the sequence of actions which need to be performed in order to access the asset record.

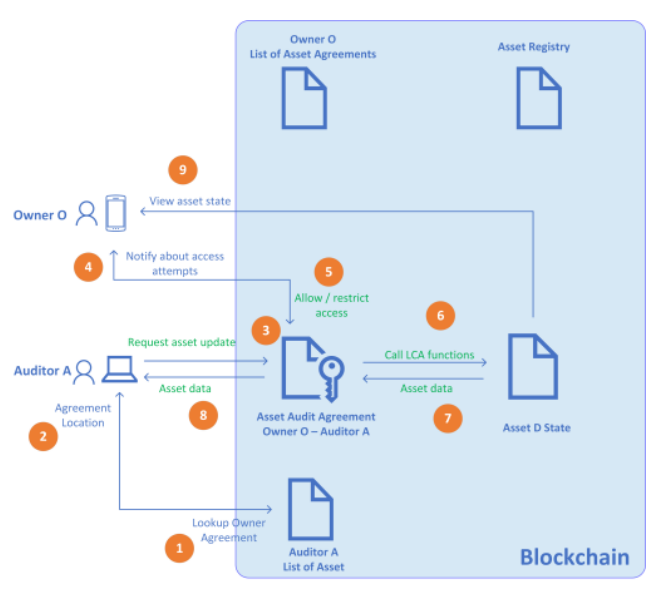

Fig. 5: Sequence to allow auditor access to the operator's asset record

Similar to the previous flow in Fig. 4, only the transactions in green will alter the state of the contracts and will be recorded on the blockchain.

\section{FUTURE WORK AND CONCLUSION}

\section{A. Application Enhancements}

Besides the underlying structure for regulating access to an asset's state record, additional off-chain modules need to be implemented in order to offer the required functionality to all blockchain participants (Operators, Auditors, 
Regulators) in a user-friendly manner. Specifically, client applications are needed which will:

- Retrieve the asset's LCA history and present it in a meaningful manner.

- Allow the asset operator to manage existing Asset State Record contract permissions.

- Allow auditors to view and update Asset State Record information.

- Allow asset operators and auditors to create, manage and view Asset Audit Agreements.

- Allow regulators to manage the operator, auditor and asset identities in the Identity Registry.

These applications can be web-based and should follow the "Distributed Application" (or "DApp") paradigm [34], and communicate with the contracts via blockchain-specific libraries (e.g. web3.js for the Ethereum blockchain [35]).

\section{B. Conclusion}

Blockchains are a powerful technology which introduces new levels of data sharing, transparency and control. Specifically in the domain of circular economy generated data, blockchains can act as an enabler for a new breed of decentralized systems and applications. Governmental environmental authorities by virtue of their role as a regulator, can leverage the benefits of blockchains while retaining a sufficient degree of control over the blockchain application. This makes a common view of all monitored assets possible, shared by all current and prospective controlling entities of assets, while at the same time, ensures that asset operators retain complete control of their asset's state record. Further, the concept of auditing an asset's location, condition and availability properties, which is important for both prospective asset operators and regulators can be introduced. For the latter part, Smart Contracts play a pivotal role towards offering granular and dynamic control of a state record. We have shown that they can be flexible enough to satisfy the main requirements for implementing and accessing asset records. As such, blockchains combined with smart contracts represent an attractive and arguably more efficient alternative to a centralized circular economy asset monitoring system for environmental regulators.

\section{REFERENCES}

[1] Ellen MacArthur Foundation, SUN and McKinsey Center for Business and Environment. Growth Within: A Circular Economy Vision for a Competitive Europe (2015)

[2] World Economic Forum. The Fourth Industrial Revolution 'is already here - and it is a matter of survival' (Press release, 26 October 2015)

[3] Ellen MacArthur Foundation. Towards the Circular Economy: Economic and Business Rationale for an Accelerated Transition (2015)

[4] O. Okorie, C. Turner, F. Charnley, M. Moreno, A. Tiwari. A review of data driven approaches for circular economy in manufacturing. Proceedings of the 18th European Roundtable for Sustainable Consumption and Production, 2017 Skiathos Island, Greece

[5] A. Pagoropoulos, D. Pigosso, T. McAloone. The emergent role of digital technologies in the Circular Economy: A review. 9th CIRP IPSS Conference: Circular Perspectives on Product/Service-Systems, 2017 Copenhagen

[6] Ellen MacArthur Foundation.Intelligent Assets: Unlocking the circular economy potential (2016)
[7] V. Salminen, H. Ruohomaa, J. Kantola. Digitalization and big data supporting responsible business co-evolution. Advances in intelligent systems and computing, vol. 498, Springer 2017

[8] M. Lider, A.Rashid. Towards Circular Economy implementation: A comprehensive review in context of manufacturing industry. Journal of Cleaner Production. 115. 10.1016/j.jclepro.2015.12.042. Springer, 2015

[9] M. Linder, M. Williander. Circular Business Model Innovation: Inherent Uncertainties. Business Strategy and the Environment. 10.1002/bse.1906. Wiley, 2015

[10] M. Lewandowski. Designing the Business Model for Circular Economy - Towards the Conceptual Framework. Sustainability Journal Vol.8 Issue 1, MDPI 2016

[11] McKinsey. The circular economy: Moving from theory to practice. McKinsey Center for Business and Environment, Special Edition, October 2016

[12] M, Laubscher, T. Marinelli, Thomas. Integration of Circular Economy in Business. 10.13140/2.1.4864.4164. Going Green Conference - CARE INNOVATION 2014, Vienna

[13] A. Pateli, G. Giaglis. A Research Framework for Analysing eBusiness models. $\quad$ EJIS. $13 . \quad 302-314$ 10.1057/palgrave.ejis.3000513. European Journal of Information Systems, November 2004

[14] J. Scott. The sustainable business: A practitioner's guide to achieving long-term profitability and competitiveness. Greenleaf Publishing, Sheffield UK, 2015

[15] K. van Renswoude, A. Wolde, D. Joustra. Circular Business Models - Part 1: An introduction to IMSA's circular business model scan. IMSA Amsterdam, April 2015

[16] D. Joustra, E. Jong, F. Engelaer. Guided choices towards a Circular Business Model. Interreg IV-B project $\mathrm{C} 2 \mathrm{C}$ BIZZ Conference, Eindhoven 2013

[17] E. de Jong, F. Engelaer, M. Mendoza. Realizing opportunities of a circular business model. DLL Group 2015.

[18] A. Jablonski. Design and Operationalization of Technological Business Models, Acta Universitatis Agriculturae et Silviculturae Mendelianae Brunensis Vol. 63 Number 3, 2015

[19] A. Parlikad, D. Mcfarlane, E. Fleisch, S. Gross. The Role of Product Identity in End of Life Decision Making. https://www.alexandria.unisg.ch/publications/21457, CAMAUTOID-WH017, 2013

[20] Ellen MacArthur Foundation. Delivering the Circular Economy. A Toolkit for Policymakers. (2015)

[21] J. De Winter. An opportunity to generate new value, recover value and mitigate risk associated with pressure on raw material availability and price volatility. Master Thesis, Univ. of Utrecht, 2014

[22] P. Lacy, D. Rosenberg, Q. Drewell, J. Rutqvist. 5 Business Models That Are Driving The Circular Economy. https://www.fastcompany.com/1681904/5-business-models-that-aredriving-the-circular-economy

[23] K. Winans, A. Kendall, H. Deng. The history and current applications of the circular economy concept. Renewable and Sustainable Energy Reviews, vol 68, issue P1, 2017

[24] H. Winkler. Closed-loop production systems - A sustainable supply chain approach. CIRP Journal of Manufacturing Science and Technology, December 2011

[25] G. Roos. Business Model Innovation to Create and Capture Resource Value in Future Circular Material Chains. Resources Journal Vol. 3 Issue 1, MDPI 2014

[26] N. Bechtel, R. Bojko, R. Volkel. Be in the loop: Circular Economy \& Strategic Sustainable Development. Master's Thesis, Blekinge Institute of Technology, Karlskrona, 2013

[27] M. Niero, A.J. Negrelli, S.B. Hoffmeyer, S.I. Olsen, M. Birkved. Closing the loop for aluminum cans: Life Cycle Assessment of progression in Cradle-to-Cradle certification levels. Journal of Cleaner Production 126, March 2016

[28] K. Christidis, M, Devetsikiotis. Blockchains and Smart Contracts for the Internet of Things. IEEE Access, Vol. 42016

[29] V. Buterin. A next-generation smart contract and decentralized application platform. https://github.com/ethereum/wiki/wiki/WhitePaper 
[30] Ethereum Foundation. Decentralized Autonomous Organization. https://ethereum.org/dao

[31] JP Morgan Chase. Quorum, a permissioned implantation of Ethereum supporting data privacy. https://github.com/jpmorganchase/quorum

[32] Solidity. A contract-oriented high-level language for implementing smart contracts. http://solidity.readthedocs.io/en/v0.4.21/
[33] B. Marino, A. Juels. 2016. Setting Standards for Altering and Undoing Smart Contracts. International Symposium on Rules and Rule Markup Languages for the Semantic Web RuleML 2016

[34] ConsenSys. Dapp Architecture Designs. https://github.com/ConsenSys/Ethereum-Development-BestPractices/wiki/Dapp-Architecture-Designs

[35] web3.js Ethereum JavaScript https://web3js.readthedocs.io/en/1.0/ 